\title{
SULFITE OXIDATION MEDIATED BY ORTHO-PHENYLENEDIAMINE / CO(II)-TETRAKIS(PARA- AMINOPHENYL)PORPHYRIN COPOLYMERS IN ACID MEDIUM
}

\author{
ROXANA ARCE I*, PAULINA MÁRQUEZ², FRANCISCO HERRERA², MARÍA J. AGUIRRE2, JULIO ROMERO'.
}

${ }^{1}$ Universidad de Santiago de Chile, Departamento de Ingeniería Química, Facultad de Ingeniería, Av. B. O'Higgins 3363, Estación Central, Santiago, Chile

${ }^{2}$ Universidad de Santiago de Chile, Departamento de Química de los Materiales, Facultad de Química y Biología, Av. B. O'Higgins 3363, Estación Central, Santiago, Chile.

(Received: June 24, 2013 - Accepted: October 2, 2013)

\begin{abstract}
The aim of this work is to study the sulfite oxidation mediated by glassy carbon electrodes modified with copolymers formed by ortho-phenylenediamine (OPD) and Co(II)-tetrakis(para-aminophenyl)porphyrin (CoTAPP). The copolymer was obtained through continuous cycling of potential of a glassy carbon electrode in acid medium containing each respective homopolymer in 1:1 feeding volume, in which the ortho-phenylenediamine concentration was $1 \cdot 10^{-1} \mathrm{M}$ and the CoTAPP concentration was $1 \cdot 10^{-4} \mathrm{M}$. The film obtained is thin, stable and contains a low amount of porphyrin in comparison to the amount in orthophenylenediamine. Its electrocatalytic activity was tested toward the sulfite oxidation. In this case, this film is more electrocatalytic than the modified electrode modified with the CoTAPP electropolymer, even though ortho-phenylenediamine is inactive in this reaction. In this work the electrocatalysis process, the morphology of the film, and the difference of electrocatalytic behavior between the ortho-phenylenediamine and cobalt porphyrin homopolymers and the orthophenylenediamine-porphyrin copolymer were studied. The morphologic studies were done using Au electrodes since the electrocatalytic activity on Au and on glassy carbon are quite similar.
\end{abstract}

Keywords: conducting polymers, modified electrodes, electrocatalysis, copolymers, Co(II)-tetrakis(para-aminophenyl)porphyrin, ortho-phenylenediamine, oxidation of sulfite.

\section{INTRODUCTION}

Sulfur dioxide is one of the most widely spread additive in winemaking. Antioxidant [1] and antimicrobial effects [2-4] account for this practically indispensable additive, not only for vinification but also for other food production [5]. Sulfur dioxide can be found free or bound to phenols, gallic acid, aldehydes, and other organic compounds [6]. On the other hand, a high level of this compound has toxic effects [7]. For that reason, its use is limited by strict amount of norms in all countries. Then, the development of analytical sensors that allow its fast and cheap determination has a great importance, for example, through electrochemical techniques [8]. Sulfur dioxide in solution can appear in different ways. The concentration of these species will mainly depend on the $\mathrm{pH}$. In $\mathrm{pH}$ conditions higher than $\mathrm{pKa}_{1}$ of the sulfur acid (see eq.1), bisulfite is present, and at $\mathrm{pH}$ values higher than $\mathrm{pKa}_{2}$ (see eq.2) only sulfite is present [9].

$$
\begin{aligned}
& \mathrm{H}_{2} \mathrm{SO}_{3} \rightleftharpoons \mathrm{H}^{+}+\mathrm{HSO}_{3} \\
& \mathrm{HSO}_{3}^{-} \rightleftharpoons \mathrm{H}^{+}+\mathrm{SO}_{3}^{2-}
\end{aligned}
$$

There are studies that show that modified electrodes are electroactive for sulfite oxidation in acid and alkaline media $[10,11]$. For example, this reaction is electrocatalized by macrocyclic complexes such as cobalt and nickel porphyrins on glassy carbon electrodes. In the case of the cobalt complex [12], the best results are obtained in basic medium because the sulfite is mainly $\mathrm{SO}_{3}{ }^{2-}$ and not $\mathrm{HSO}_{3}^{-}$.

Figure 1 shows one of the monomers i.e. the porphyrin monomer. Both monomers, CoTAPP and OPD have amino groups that allow solubility in aqueous acid medium and also allow electropolymerization by oxidation to generate a radical cation.

Ortho-phenylenediamine (derived from aniline) has received considerable attention as electropolymer (polyOPD). This polyOPD polymer forms a conducting film on the surface of different electrodes and it can be electrochemically synthesized on acid dissolutions. Its voltammetric response shows a single reversible redox couple [13]. On the other hand, the polymer is stable in acid, neutral and basic dissolutions and exposed to air [14]. Electrochemical techniques such as in situ FTIR spectroscopy [14-16], Raman resonance spectroscopy [17], and other methods, have been used to study the electropolymerization mechanism, to characterize the polymer film and to discover different applications of polyOPD [18]. Also, it is possible to polymerize porphyrins of different transition metals [19-21], one of them being
Co(II)-tetrakis(para-aminophenyl)porphyrin [22-29] on different electrodic surfaces. This polymer shows electrocatalytic activity on several interesting redox reactions [22-29] such as sulfite oxidation. However films are fragile, unstable and very thin [30]. Furthermore, the copolymerization between ortho-phenylenediamine and $\mathrm{Co}(\mathrm{II})$-tetrakis(para-aminophenyl)porphyrin, that forms a random copolymer [41], results in a good combination of stability and electrocatalytic activity and could be an interesting material to prove the electro-oxidation of sulfite.

In fact, a CoTAPP/OPD copolymer and its respective homopolymers are electrochemically prepared and compared toward the sulfite electro-oxidation. The copolymer shows an enhanced activity compared to the porphyrin film in spite of the other monomer that is inactive toward the reaction.

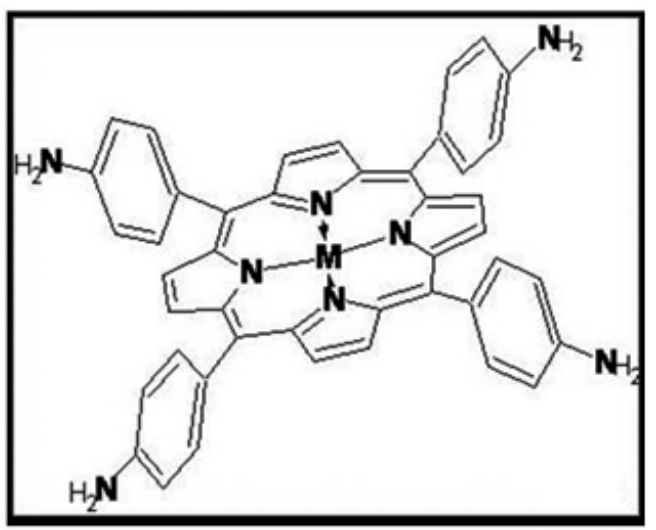

Figure 1. Structural formula of $\mathrm{Co}(\mathrm{II})$-tetrakis(para-aminophenyl) porphyrin CoTAPP. M represents Co.

\section{EXPERIMENTAL}

CoTAPP was obtained from Organix (http://www.essex.ac.uk/guest/ organix/). OPD was purchased from Aldrich Chemical \& Co. These both products were used without further purification. Glassy carbon (GC) electrodes were purchased from $\mathrm{CH}$ Instruments, Inc. and gold electrodes $\mathrm{Au}$ ) were purchased from Aldrich. Electrochemical experiments were performed in a three-compartment glass cell, where glassy carbon $\left(\mathrm{A}=0.071 \mathrm{~cm}^{2}\right)$ and $\mathrm{Au}$ $\left(\mathrm{A}=1.5 \mathrm{~cm}^{2}\right)$ were used as working electrodes. A saturated $\mathrm{Ag} / \mathrm{AgCl} / \mathrm{KCl}$ (sat) 
was used as reference electrode - so all the potentials that are quoted in this work are related to this reference electrode - and a Pt coil was used as a counter electrode. The glassy carbon electrodes were polished with $0.25 \mu \mathrm{m}$ alumina before each experiment. The copolymer films were grown on the electrode by potentiodynamical cycles between -0.6 and $1.0 \mathrm{~V}$ during 15 cycles at a scan rate $0.05 \mathrm{~V} \cdot \mathrm{s}^{-1}$ in $0.1 \mathrm{M} \mathrm{HCl}$ aqueous solution containing $0.1 \mathrm{mM} \mathrm{Co}(\mathrm{II})-$ tetrakis(para-aminophenyl)porphyrin and $0.1 \mathrm{M}$ OPD (1:1 in volumetric feed). The solution was purged with nitrogen (ultra pure grade) during each polymerization. After polymerization, the modified electrode was rinsed with deionized and distilled water.

Same conditions were used when homopolymerizations were done, but the range of potentials was between $-0.6 \mathrm{~V}$ and $1.0 \mathrm{~V}$ for CoTAPP and between $0.0 \mathrm{~V}$ and $0.8 \mathrm{~V}$ for OPD. All the experiments were carried out at room temperature and under nitrogen atmosphere. Electrochemical measurements were performed using a CHI 604 Potentiostat, along with PC. Characterization performed by Scanning Electron Microscopy (SEM) in a Carl Zeiss EVO MA 10 , operated at $7.00 \mathrm{kV}$.

Electrochemical measurements of the sulfite electro-oxidation were performed in $12 \%$ ethanol aqueous solution containing $0.1 \mathrm{M} \mathrm{NaCl}, 0.1 \mathrm{M}$ $\mathrm{NaOH}\left(\mathrm{pH} 10\right.$ measured by a $\mathrm{pHmeter}$ ) and $0.1 \mathrm{mMNa}_{2} \mathrm{SO}_{3}$, cycling the potential between 1.0 and $-1.0 \mathrm{~V}$ at a scan rate of $0.1 \mathrm{~V} \cdot \mathrm{s}^{-1}$. All these electrocatalytic studies were carried out on bare and on modified with polyOPD, polyCoTAPP and copolymer glassy carbon or Au electrodes. Controlled potential electrolysis $(0.6 \mathrm{~V}, 0.7 \mathrm{~V}, 0.8 \mathrm{~V}, 0.9 \mathrm{~V}$ and $1.0 \mathrm{~V}$ during $1200 \mathrm{~s})$ toward sulfite electrooxidation were performed by copolymer modified electrodes in which a sample of the resulting solution was added to excess dilute $\mathrm{HCl}$, then it was added a few drops of barium chloride. The formation of a white precipitate finely divided, insoluble, indicated the presence of sulfate.

\section{RESULTS AND DISCUSSION}

\section{Electrochemical Characterization of homo and copolymers}

Figure 2 shows the $15^{\text {th }}$ potentiodynamic cycle corresponding of the obtaining of the copolymer and the corresponding homopolymers at $0.05 \mathrm{~V} \cdot \mathrm{s}^{-1}$ in $0.1 \mathrm{M} \mathrm{HCl}$. The inset of Figure 2 shows the redox couple of $\mathrm{Co}(\mathrm{III}) / \mathrm{Co}$ (II) where the Co (II) oxidation appears at $0.61 \mathrm{~V}$ (III) and the reduction appears at $0.43 \mathrm{~V}$ for polyCoTAPP (III) [41]. The position of these redox peaks is shown also in the Figure 2 (polyCoTAPP) where they do not appear because of the scale of the Figure. The characteristic redox couple phenazine/phenacyl [13] is not observed in polyOPD because it appears at more negative potentials than the potential window used. The oxidation waves observed at the positive limits of the potential correspond to the oxidation of amino groups, which originates the radical cations responsible of polymerization. The positive limit changes for polyCoTAPP and polyOPD because the electronic density of the amino groups changes depending on the nature of the compound. In fact, at $1.0 \mathrm{~V}$ polyOPD sobreoxidizes and loses activity. In the case of the copolymer, the peak corresponding to the Co(II) oxidation does not appear, probably masked by the phenazine/phenacyl redox couple ( $I_{a}$ e I ), while the cathodic process $\mathrm{Co}(\mathrm{III}) / \mathrm{Co}(\mathrm{II})$, observed at $0.28 \mathrm{~V}$ (III), increased in current, if it is compared to the process observed in polyCoTAPP, and shifted to more negative potentials. It is interesting to point out that the porphyrin changes the potential of its redox processes when its neighborhood changes because of the covalent linkage between OPD and CoTAPP [41], indicating the electronic communication between the two different molecules. Also a shifting of the potential is taking place for the amino groups (II) of the copolymer compared to the two homopolymers [42].

Figure 3 shows the effect of scanning rate for the phenazine/phenacyl redox couple on the copolymer, at ca. $-0.5 \mathrm{~V}$. The current increases with the scanning rate (v) and the slope of Ep versus $\log \mathrm{v}=1$ (not shown) indicating that the film is conductive and the redox process has kinetic control.

\section{Morphological Characterization of homo and copolymers}

SEM image of bare gold (Figure 4A) shows a clean and plain surface, which was used as a reference since it is the used substrate. Figure 4B shows the SEM image of polyOPD growth on the gold surface. It can be observed a polymer that forms $2 \mu \mathrm{m}$ wide well defined filaments forming an irregular or not homogeneous film on the electrode surface.

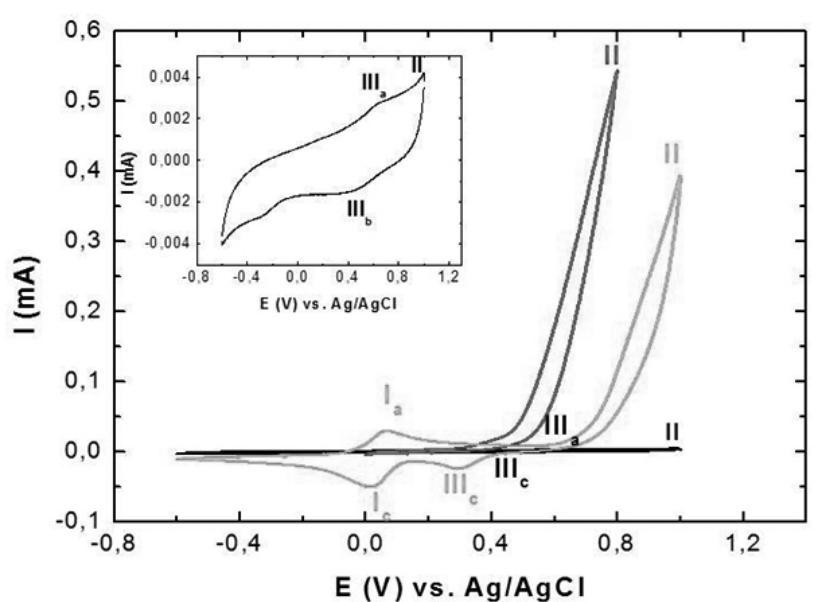

Figure 2. Comparative voltammetric profiles obtained during the electropolymerization of CoTAPP (line black), OPD (line red) and copolymer (line green) on glassy carbon electrode in $0.1 \mathrm{M} \mathrm{HCl} . \mathrm{v}=50 \mathrm{mV} \cdot \mathrm{s}^{-1}$. Cycle 15. The insert shows the voltammetric profile of polyCoTAPP in the same conditions outlined above.

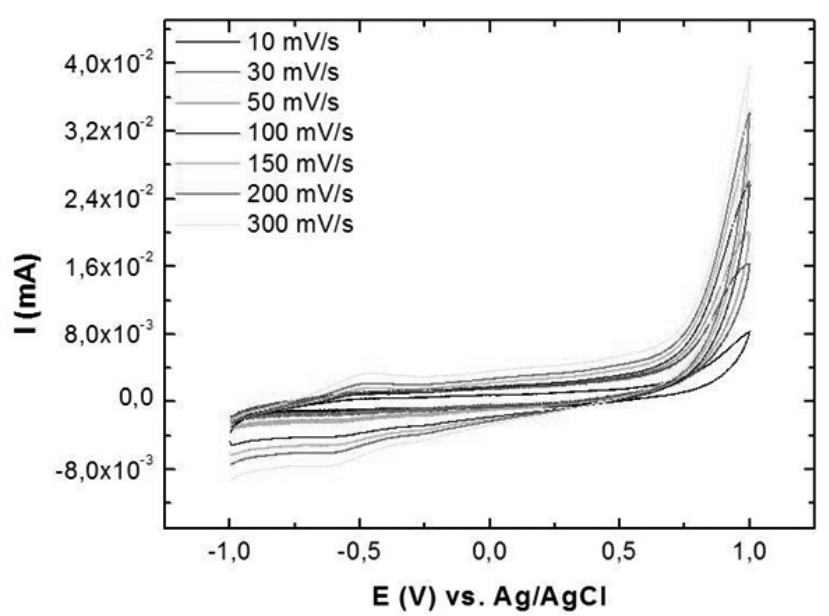

Figure 3. Profiles of effect of the scanning rate of glassy carbon electrode modified with copolymer for the electro-oxidation of sulfite. $\mathrm{v}$ : $100 \mathrm{mV} \cdot \mathrm{s}^{-1}$. Cycle 1.
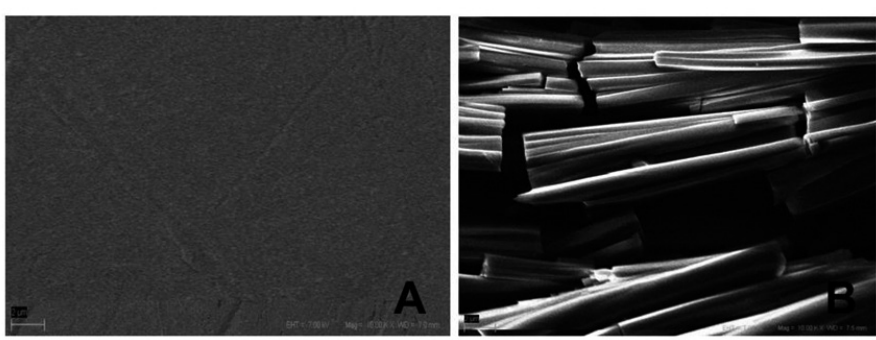

Figure 4. Scanning electron micrograph of gold electrode. $10 \mathrm{KX}$ Magnification (A), gold electrode modified with polyOPD $10 \mathrm{KX}$ Magnification (B).

Furthermore, figures 5A and 5B show the CoTAPP polymer on gold. In this case, the polymer forms a homogeneous rough film that practically covers the whole electrode surface. The thickness of this film was measured by a $45^{\circ}$ cut. The thickness of the polyCoTAPP film was $500 \mathrm{~nm}$. Figures 5C y 5D show the growth of the copolymer film. The surface appears completely covered by a porous film. 

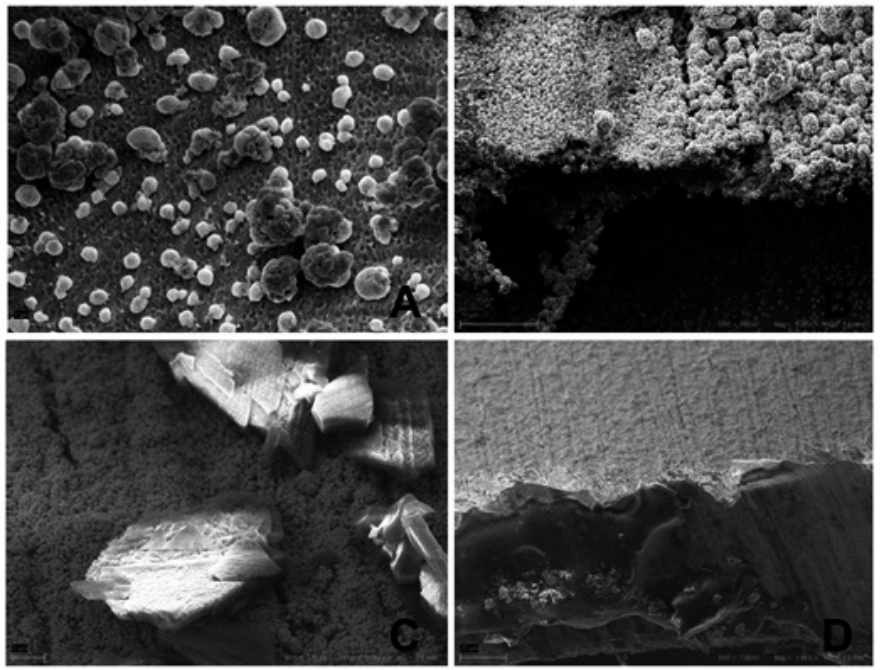

Figure 5. Scanning electron micrographs of glassy carbon electrode modified with polyCoTAPP $25 \mathrm{KX}$ Magnification (A), polyCoTAPP $5.00 \mathrm{KX}$ Magnification (B) in a cross-section, copolymer 10 KX Magnification (C) and copolymer 1.49 KX Magnification (D) in a cross-section.

This result shows that for some reason, which can be ascribed to the solubility of oligomers that are formed on the electrode-electrolite interphase, the polyCoTAPP precipitation is favored and not the polyOPD precipitation. It is not clear if the oligomers that are firstly formed are the porphyrin ones or the cobalt porphyrins, which are bond to OPD. Nevertheless, the similarity of the final polymer between the polyCoTAPP homopolymer and the copolymer indicates that the principal monomer on both cases is porphyrin. In this case, OPD acts as a support that allows the porphyrin oligomers, which are firstly formed for bonding to OPD, to acquire more conductivity than its monomers by themselves. The resolution of the morphology measured by SEM for the polymer is higher than the one for the homopolymers, indicating, this time, a direct method that has higher conductivity.

\section{Electrocatalytic Characterization of homo and copolymers}

\section{Electro-oxidation of sulfite}

Figure 6 shows the comparison between the three modified electrodes toward the sulfite electro-oxidation. The copolymer is the most active, since it promotes the oxidation at a lower positive potential than the polyCoTAPP, and with higher current, too. The polyOPD modified electrode is inactive for this reaction. In all cases, the product of the oxidation is sulfate. Sulfate was detected after electrolysis by precipitation with $\mathrm{Ba}^{2+}$, where barium sulfite and sulfate precipitate. The precipitate is separated and $\mathrm{HCl}$ is added, which allows the barium sulfite redissolution. The remaining precipitate is sulfate. These species are formed through an irreversible oxidation process [43, 44].

It is noticeable the high electrocatalytic activity of the copolymer toward sulfite oxidation, which should be given by the presence of Co porphyrin on its structure. According to the feeding during the copolymerization process, the porphyrin content in the copolymer should be 1000 times lower than the OPD content, but according to the charge relation between the phenazine/phenacyl redox couple and the Co redox couple, the relation for the composition is $4: 1$ (OPD: CoTAPP). The linkage between both monomers allows the shifting of the potential for sulfite oxidation.

On the other hand, the modified electrodes with the copolymer and polyCoTAPP are stable in presence of sulfite after 10 repetitive potential cycles, since their I/E profiles remain constant. However, the modified electrode with polyOPD is less stable because a decrease in the current during the 10 repetitive cycles takes place.

\section{CONCLUSIONS}

The simultaneous electro-oxidation of OPD and CoTAPP generates a copolymer on glassy carbon or gold surface electrodes. This copolymer is a better electrocatalyst for sulfite oxidation than polyCoTAPP in spite of the inactivity of the other monomer, OPD. In fact, the copolymer shifts the oxidation wave corresponding to the oxidation of sulfite to sulfate to more negative potentials. The copolymer is stable to the continuous cycling of potential in the presence or not of sulfite in the solution whereas polyOPD is very unstable.

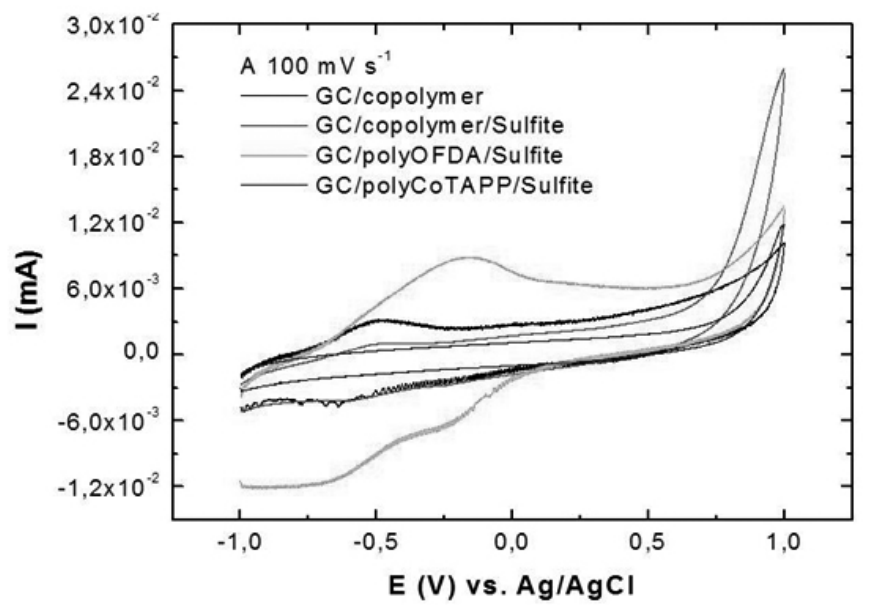

Figure 6. Profiles of comparative voltammetry of glassy carbon electrode modified with polyCoTAPP, polyOPD and copolymer for the electro-oxidation of sulfite at a scan rate of $100 \mathrm{mV} \cdot \mathrm{s}^{-1}$. Cycle 1 .

\section{ACKNOWLEDGEMENT}

In memory of Dra. Elsa Abuin Saccomano.

This paper was financially supported by Fondecyt Postdoctoral No 3130594 project. M. J. A. acknowledges Fondecyt 1120071 project.

\section{REFERENCES}

1. C. S. Ough and E. A. Crowell, Journal of Food Science, 52, 386 (1987).

2. L. A. Sayavedra-Soto and M. W. Montgomery, Journal of Food Science, 51, 1531 (1986).

3. F. W. Beech, L. F. Burroughs, C. F. Timberlake, and G. C. Whiting, Bulletin de l'OIV, 52, 1001 (1979).

4. P. Romano and G. Suzzi, WineMicrobiology and Biotechnology, Harwood Academic Publishers, Chur, Switzerland, 1993.

5. L. C. Schroeter, Sulfur Dioxide, Applications in Foods, Beverages and Pharmaceuticals, Pergamon Press Inc., Oxford, UK, $1^{\text {st }}$ edition, 1966.

6. I.Hornsey, The Chemistry and Biology of Winemaking, Thomas Graham House, 2007.

7. H. J. Schwartz, Journal of Allergy and Clinical Immunology, 71, 487 (1983).

8. C. Montes, J. H. Vélez, G. Ramírez, M. Isaacs, R. Arce, and M. J. Aguirre., The Scientific World Journal, 2012, doi: 10.1100/2012/168148 (2012).

9. R. David, Handbook of Chemistry and Physics $73^{\mathrm{a}}$ Ed. CRC Press, Inc. Florida, 8-37, 2000.

10. H. Zhou, W. Yang and C. Sun, Talanta, 77, 366 (2008).

11. Y. Tolmachev and D. Scherson, Electrochim. Acta, 49, 1315 (2004).

12. R. Carballo, V. Campo Dall'Orto, A. Lo Balbo and I. Rezzano, Sensors and Actuators B, 88, 155 (2003).

13. H-P. Dai, Q-H. Wu, S-G. Sun and K-K. Shiu, J. Electroanal. Chem., 456, 47 (1998).

14. K. Ogura, M. Kokura, J. Yano and H. Shigi, Electrochim. Acta, 40, 2707 (1995).

15. D. Wei and H-H. Wu, Chem. J. Chin. Univ., 16, 1761; 6, 423 (1995; 1994).

16. X-Q. Lin and H-Q. Zhang, Electrochim. Acta, 41, 2019 (1996).

17. L. Wu, J. Luo and Z. H. Lin, J. Electroanal. Chem., 417, 53 (1996).

18. K. Martinusz, E. Czirok and G. Inzelt, J. Electroanal. Chem., 379, 437 (1994).

19. B. A. White and R. W. Murray, J. Electroanal. Chem., 189, 345 (1985).

20. M. A. Riquelme, M. Isaacs, M. Lucero, E. Trollund, M. J. Aguirre, J. Canales, J. Chil. Chem. Soc., 48, 2 (2003). 
21. P. Bravo, F. Isaacs, G. Ramírez, F. Armijo, M. Isaacs, M. J. Aguirre, C. Zamora, E. Trollund, J. Chil. Chem. Soc., 50, 671 (2005).

22. W. Cheuquepán, D. Villagra, M. I. Azócar, G. Ramírez, Y.Y. Chen, B. Matsuhiro, L. Mendoza, M. Isaacs, M. J. Aguirre, J. Chil. Chem. Soc., $\mathbf{5 5}, 253(2010)$.

23. G. Ramírez, G. Cornejo, M. Lucero, A. Riquelme, I. Azócar, F. Armijo, M. J. Aguirre, E. Trollund, J. Chil. Chem. Soc., 49, 261 (2004).

24. P. Dreyse, G. Ramírez, A. Riquelme, M. Isaacs, J. Chil. Chem. Soc., 51, 2 (2006). A. N. K. Lau and L. L. Miller, J. Am. Chem. Soc., 105, 5271 (1983).

25. S. Chao, J. L. Robbins and M. S. Wrighton, J. Am. Chem. Soc., 105, 181 (1983).

26. F. Armijo, Tesis Grado de Doctor en Química, Facultad de Química y Biología, Universidad de Santiago de Chile, (2004).

27. X. Ren and P. G. Pickup, J. Electroanal. Chem., 365, 289 (1994).

28. A. W. Bott, Current Separations, 19, 71 (2001).

29. F. B. Kaufman and M. B. Engler, J. Am. Chem. Soc., 101, 547 (1979)

30. S. Griveau, V. Albin, T. Pauporté, F. Bedioui and J. Zagal, J. Mater. Chem., 12, 225 (2002).

31. C. M. A. Brett, A. M. Oliveira, Electrochemistry Principles, Methods and Aplications, In: Oxford University Press Inc., New York, 253, 1993.

32. W. C. Chen, T. C. Wen and A. Gopalan, J. Electrochem. Soc., 148, E427
(2001).

33. K. Yamamoto and D. Tanachi, Macromol. Chem. Phys., 201, 649 (2000).

34. J. Y. Heras, A. F. F. Giacobone and F. Battaglini, Talanta, 71, 1684 (2007).

35. R. M. Latonen, C. Kvarnstoma and A. Ivaska, Electrochim. Acta, 44, 1933 (1999).

36. Y. W. Chen-Yang, J. L. Li, T. L. Wu, W. S. Wang and T. F. Hon, Electrochim. Acta, 49, 2031 (2004).

37. H. Tang, A. Kitani and S. Ito, Electrochim. Acta, 42, 3421 (1997).

38. S. Bilal and R. Holze, Electrochim. Acta, 52, 5346 (2007).

39. S. Bilal and R. Holze, Electrochim. Acta, 52, 1247 (2006).

40. M. Lucero, M. Riquelme, G. Ramírez, M. C. Goya, A. González Orive, A. Hernández Creus, M. C. Arévalo and M. J. Aguirre, Int. J. Electrochem. Sci., 7, 234 (2012).

41. R. Arce, R. del Río, D. Ruíz-León, J. Velez, M. Isaacs, María A. del Valle and María J. Aguirre, Int. J. Electrochem. Sci., 7, 11596 (2012).

42. P. Ardiles, E. Trollund, M. Isaacs, F. Armijo, J. C. Canales, M. J. Aguirre and M. J. Canales, J. Molecular Catalysis A: Chemical, 165, 173 (2001).

43. S-M. Chen and Y-L. Chen, J. Electroanal. Chem., 573, 277 (2004).

44. M. Lucero, G. Ramírez, A. Riquelme, I. Azócar, M. Isaacs, F. Armiijo, J. E. Forster, E. Trollund, M. J. Aguirre and D. Lexa, J. of Molecular Catalysis A: Chemical, 221, 71 (2004). 